\title{
Performance of preschool children on three discrimination shifts'
}

DONALO J. DICKERSON2 INSTITUTE OF CHILD BEHAVIOR AND DEVELOPMENT, UNIVERSITY OF IOWA

After learning an initial two-dimensional discrimination, preschool children were given reversal (RV), intradimensional (ID), and extradimensional (ED) shifts. RV and ID shifts were learned faster than ED shifts, a finding contradictory to the mediational theory of Kendler \& Kendler (1962).

A mediational theory of discriminative learning in which the mediational process is more readily available to adults than to young children and animals has been presented by Kendler \& Kendler (1962). Lending support to this formulation are the results of experiments comparing reversal (RV) and extradimensional (ED) shift performance. While it has been found consistently that adult Ss learn RV shifts faster than ED shifts (Isaacs \& Duncan, 1962; Kendler \& D'Amato, 1955), the results of other experiments suggest that young children (below approximately 6 yr.) learn ED shifts faster than RV shifts (Kendler \& Kendler, 1959; Kendler, Kendler, \& Wells, 1960; Marsh, 1964; Tighe \& Tighe, 1965). Eimas (1965) recently has made a criticism which bears upon the methodology of these latter four studies. All have employed ED-shift procedures in which the relevant dimension of Problem 1 was made constant within trials in Problem 2. Perhaps a mediational response is not elicited strongly by a stimulus dimension unless the dimension is variable within trials. An RV-ED shift difference in favor of mediational theory might not be expected unless this condition were met. The suggestion is that the conclusion of a developmental change in the mediational process may not be warranted.

The present experiment compares RV and ED shift performances in preschool children. An ED-shift procedure is used in which the relevant dimension of Problem 1 becomes variable-irrelevant in Problem 2. An intradimensional (ID) shift condition is included. A comparison of ID and $\mathrm{ED}$ shift performance provides the more appropriate test of mediational theory since sources of instrumental response transfer are eliminated.

\section{Method}

The discriminative stimuli consisted of three-dimensional objects differing in form (triangle, circle, square, T) and color (red, green, yellow, blue). Each of these stimuli measured 2-3/4 in. in both length and width and $1 / 2$ in. in height. The stimuli were mounted on $3-3 / 4 x$ $3-3 / 4$ in. gray blocks. Each block was halved along the frontal plane with the two halves joined by a hinge. The interior of each block contained a marble well which was exposed upon displacement of the top half of the block. The two stimuli to be discriminated were placed 10 in. apart on a gray turntable. A screen in the center of the turntable prevented the $\mathrm{S}$ from observing the $\mathrm{E}$ as each trial was prepared.

All Ss were given two-choice, simultaneous discriminations in Problem 1 involving two component values of each of the dimensions on which the stimuli varied. One of these dimensions was relevant and the other was variable-irrelevant. The rewarded component of the relevant dimension was designated at random and this component was paired with each component of the irrelevant dimension an equal number of times on the right and left. A marble served as the reward stimulus. Correct responses also were rewarded with "good" and incorrect responses were punished with "no." Correction of erroneous responses was not allowed. Position of reward was determined by a Gellermann series.

Problem 2 of the RV shift involved the same stimuli as Problem 1. However, a response to the previously negative component now was rewarded. In Problem 2 of the ID and ED shifts, two new values on both the color and form dimensions were introduced. The relevant and irrelevant dimensions were unchanged in the ID shift, while in the ED shift the relevant Problem 1 dimension became irrelevant and vice-versa. Color was relevant in Problem 2 for half the Ss in each shift group, and form was relevant for the remaining Ss.

The Ss were 48 children attending the Institute of Child Behavior and Development Preschool Laboratories and the Iowa City Parents' Cooperative Preschool. Ss were assigned at random to the six shift-by-dimension groups ( $8 \mathrm{Ss} /$ group). CAs ranged from 45 to $67 \mathrm{mos}$. with a mean of 56.1 mos.

Three experimental sessions were required for each S. These sessions generally were separated by a period of two days. On the first day, pretraining was given on a discrimination involving a pair of "junk" stimuli. Pretraining was continued until a criterion of 10 consecutive correct responses was reached. On the second day, each $\mathrm{S}$ again was brought to a criterion of 10 consecutive correct responses on the pretraining problem. Immediately after reaching criterion, the S began training on Problem 1 and continued until a criterion of 10 consecutive correct responses was reached. If $S$ had not attained this criterion at the end of 40 trials, a special training procedure was instituted to assure learning. Without mentioning the color or form of the stimuli, the E showed the two positive stimulus objects to the $\mathrm{S}$ and said, "These blocks are right. The marble is always in these blocks." 
The $\mathbf{E}$ then presented the two negative stimulus objects and said, "These blocks are wrong. The marble is never in these blocks." Following this special training, all Ss began criterion performance within 15 trials. On the final day, each S relearned Problem 1 to a criterion of 10 consecutive correct responses. Then training was begun immediately on Problem 2 and continued until a criterion of 20 consecutive correct responses was reached, or for a total of 80 trials.

\section{Results}

Means of the error scores for the three shift groups are given in Table 1. Each error score was either the number or errors to criterion or the number of errors to the end of training ( 80 trials).

Since the subgroup variances were extremely heterogeneous, an extension of the analysis of variance by ranks was used. A 3 by 2 factorial analysis of variance of this type was performed on the error scores. In this analysis, the factors were type of shift and relevant dimension in Problem 2. The Type-of-Shift main effect was significant, $\mathrm{H}=14.53, \mathrm{df}=2, \mathrm{p}<.001$. The RelevantDimension main effect was not significant, $H=0.71$, $\mathrm{df}=1, p>.30$. In addition, the interaction of these two factors was nonsignificant, $\mathrm{H}=1.13, \mathrm{df}=2, \mathrm{p}>.50$. In order to determine the source of the significant Typeof-Shift main effect, three Mann-Whitney U tests were performed. The results of these tests indicated that, while the RV and ID shifts did not differ significantly in difficulty, both were significantly less difficult than the ED shift ( $p<.002$ for both tests).

Table 1. Means of Error Scores for the Three Shift Groups

\begin{tabular}{lccc} 
Shift & Form & Color & Total \\
\hline RV & 1.38 & 1.25 & 1.32 \\
ID & 1.00 & 5.25 & 3.12 \\
ED & 10.25 & 22.38 & 15.50 \\
\hline
\end{tabular}

\section{Discussion}

The results of this experiment strongly suggest that preschool children make mediational responses to the relevant dimension of discrimination problems, and that they transfer these responses to subsequent problems. In addition, Shepp \& Eimas (1964) have reported data showing that even rats learn ID shifts faster than ED shifts. Thus, it appears unnecessary to assume a developmental change in the discrimination learning process in order to explain the disparate findings, Other experiments with young children may have employed ED-shift procedures which have served to minimize the interfering effects of mediating responses.

\section{References}

Eimas, P. D. Comment: Comparisons of reversal and nonreversal shifts. Psychon. Sci., 1965, 3, 445-446.

Isaacs, I. D., \& Duncan, C. P. Reversal and nonreversal shifts within and between dimensions in concept formation. $J$. exp. Psychol., 1962, 64, 580-585.

Kendler, H. H., \& D'Amato, M. F. A comparison of reversal shifts and nonreversal shifts in human concept formation behavior. $J$. exp. Psychol., 1955, 48, 165-174.

Kendler, H. H., \& Kendler, T. S. Vertical and horizontal processes in human problem solving. Psychol. Rev., 1962, 69, 1-18.

Kendler, T. S., \& Kendler, H. H. Reversal and nonreversal shifts in kindergarten children. J. exp. Psychol., 1959, 58, 56-60.

Kendler, T. S., Kendler, H. H., \& Wells, D. Reversal and nonreversal shifts in nursery school children. J. comp. physiol. Psychol., 1960, 53, 83-88.

Marsh, G. Effect of overtraining on reversal and nonreversal shifts in nursery school children. Child Develpm., 1964, 35, 1367-1372.

Shepp, B. E., \& Eimas, P. D. Intradimensional and extradimensional shifts in the rat. $J$. comp. physiol. Psychol, 1964, 57, 357-361.

Tighe, L. S., \& Tighe, T. J. Overtraining and discrimination shift behavior in children. Psychon. Sci., 1965, 2, 365-366.

\section{Notes}

1. This paper was prepared while the author was on a predoctoral research fellowship supported by George Peabody College for Teachers. The author is indebted to Gordon N. Cantor, Charles E. Clifton, and Dee W. Norton for counsel during the experiment. 2. Now at the University of Connecticut. 